\title{
Krebsregister
}

\section{Regionale Heterogenität erschwert den bundesweit raschen Aufbau}

\author{
Sie sollen die Versorgung der Krebspatienten verbessern: Klinische \\ Krebsregister werden Realität. Doch wann? Der Teufel steckt im Detail: \\ Noch gibt es viele offene Fragen zu klären.
}

$\mathrm{M}$ ehr Daten zur Diagnose und Behandlung von Krebserkrankungen sowie über den Therapieverlauf - Krebspatienten sollen künftig so schnell wie möglich von aktueller Forschung bei der Behandlung profitieren können. Ermöglichen sollen dies klinische Krebsregister: Doch noch gibt es viele Baustellen bei deren Aufbau. Das ist ein Fazit des Cognomed Branchentreff Onkologie am 19. April 2013 in Berlin.

Bislang fehlten bundesweit einheitliche Strukturen der Datenerfassung, sagte Dr. Monika Klinkhammer-Schalke von der Arbeitsgemeinschaft Deutscher Tumorzentren. Ein erstes Treffen, um diese zu definieren, habe im März unter Leitung des Bundesgesundheitsministeriums stattgefunden. Eine besondere Aufgabe beim Aufbau dieser Strukturen: Die Daten müssten über Ländergrenzen hinweg vergleichbar sein. Dauerhaftes Ziel sei es schließlich, das Überleben der Patienten zu verbessern, so Klinkhammer-Schalke. Und das könne nur gelingen, wenn bekannt ist, wie sich die Versorgungslage aktuell bundesweit darstelle.

Das Krebsfrüherkennungs- und -registergesetz ist im April in Kraft getreten. Damit haben die Länder den Auftrag erhalten, bundesweit klinische Krebsregister aufzubauen. Das Saarland hatte bereits vergangenes Jahr gefordert, dass die Register in drei Jahren fertig sein sollen. Dem Gesetzestext zufolge fehlen in Deutschland noch etwa 30 solcher Register. „Aber wir fangen beim Aufbau nicht bei Null an“, betonte KlinkhammerSchalke. Länder wie Bayern, Brandenburg und Mecklenburg-Vorpommern haben bereits entsprechende Strukturen. Doch auch diese Länder müssen nachsit- zen: Denn bislang sind diese völlig unterschiedlich organisiert, kritisieren Gesundheitsexperten. Darüber hinaus müsse das Einzugsgebiet definiert werden, betonte Klinkhammer-Schalke. Etwa ein bis vier Millionen Einwohner pro Krebsregister seien geplant. Niedergelassene Onkologen und Kliniken sollten dann alle Schritte zur Behandlung eines Krebsfalls an das für sie zuständige Register melden. Dieses werde dann die Daten auswerten und an die Ärzte vor Ort zurückgeben. Mehrfachdokumentationen sollen auf diese Weise vermieden werden.

\section{Investitionsfonds von acht}

Millionen Euro für die Länder

Ein Knackpunkt beim Aufbau der klinischen Krebsregister seien die Datenschutzregelungen. Bereits seit 2008 führe man Gespräche mit Datenschützern.

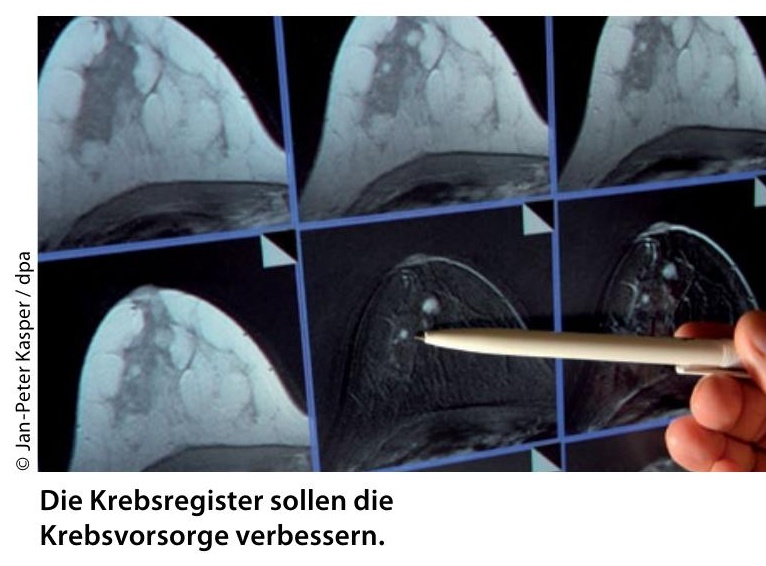

Die Konferenz der Datenschutzbeauftragten der Länder hatte erst kürzlich eine sorgfältigere Verschlüsselung der Krebsregisterdaten gefordert.

Für den Aufbau der klinischen Krebsregister soll für die Länder ein Investitionsfonds mit acht Millionen Euro zur Verfügung stehen. Die Bundesländer werden selbst 800.000 Euro beisteuern, 7,2 Millionen Euro wird die Deutsche Krebshilfe spenden. Sunna Gieseke
Hamburg startet jetzt mit Diagnose- und Therapietransparenz

Startschuss für das klinische Krebsregister in Hamburg: Gesundheitsbehörde, Ärztekammer, Krebsgesellschaft, Krankenhausgesellschaft, KV, gesetzliche und private Krankenversicherungen haben eine gemeinsame Erklärung unterzeichnet, um eine vergleichbare Datengrundlage zur Beurteilung von Erstdiagnose, Therapie, Verlauf und Ergebnis bei Krebspatienten in Hamburg zu erhalten.

„Wir schaffen damit Transparenz darüber, wo und mit welchen Methoden Krebs erfolgreich behandelt wird", sagte Gesundheitssenatorin Cornelia Prüfer-Storcks (SPD). Die Ärzte erwarten, dass die onkologische Versorgung in der Stadt profitieren wird, wie Hausarzt Klaus Schäfer, Vizepräsident der Ärztekammer, betonte. Denn die Informationen sollen regelmäßig ausgewertet und bei onkologischen Qualitätskonferenzen interpretiert werden.

In Hamburg erhalten jährlich 10.000 Menschen die Diagnose Krebs, fast 5.000 sterben jedes Jahr daran. Die gesetzliche Krankenversicherung finanziert zu 90\% den Betrieb der klinischen Krebsregister. Den Rest tragen hauptsächlich die Länder. Hamburg hat in den Etats für 2013 und 2014 jeweils 500.000 Euro dafür vorgesehen. 